\title{
Comments on Rozas
}

\section{Krister Bykvist}

Department of Philosophy, Stockholm University and Institute for Futures Studies, krister.bykvist@philosophy.su.se

Early View publication date: 1 September 2021

DOI: http://dx.doi.org/10.5324/eip.v15i2.4083

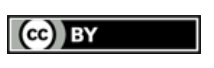

This is an open access article distributed under the terms of the Creative Commons Attribution 4.0 International License, which permits unrestricted use, distribution, and reproduction in any medium, provided the original author and source are credited.

As Rozas points out, we seem to have different intuitions about when the interests of contingent future people can outweigh those of already existing ones. In Happy Child the parents have no reason to create the child because the positive wellbeing of the possible child seems not to outweigh the loss in wellbeing for the parents, even though the child's positive wellbeing is greater than the combined losses of the parents. In Wretched Child, by contrast, it seems that the negative wellbeing of the possible child does outweigh the gains for the parents and that the parents therefore should not create the child. How can the wellbeing of the happy child matter so little when the wellbeing of the wretched child matters a lot? Rozas argues that the intuitively satisfactory answer to this question is given by what he calls the 'Asymmetry between Positive and Negative Value' ('Asymmetry', for short), which states that 'given a correlation between the intensity and the duration of a value and a disvalue, disvalue outweighs value and thus should be given greater consideration when assessing outcomes' (Rozas 2021, page 45). The wellbeing of the possible child in Wretched Child matters less than the wellbeing of the parents because the possible child would have negative wellbeing, which, according to Asymmetry, would count more than the gains in positive wellbeing for the parents. The explanation of why Asymmetry would say that they have no reason to create the happy child is less clear. We are told that views that satisfy Asymmetry

do not need to accept that we should bring into existence The Happy Child. Fully Asymmetrical Views, as well as a number of Weighted Asymmetrical Views such as the Deontological or Satisficing ones we have considered, would reject that we have reasons to create The Happy Child in any case. That is, they would accept this even under an unrealistic idealizing condition according to which bringing the Happy Child into existence would not cause any disvalue at all. Other Asymmetric Views would be compatible with accepting the creation of The Happy Child only under this unrealistic condition. But they would reject it in the real world, where in all likelihood the creation of the Happy Child, as described, would come at the cost of greater suffering, and such suffering would not be compensated by the increase in positive value. (Rozas 2021, page 46) 
It is clear that fully asymmetrical views that give no weight to positive value would say that the parents have no reason to create a uniformly happy child. But it less clear why deontological and satisficing asymmetrical views would say so. Indeed, it is unclear exactly what these views say. Rozas does not give us any definitions of these views. As I understand him, and here I expand on what he says, these views claim that, when we assess the value of outcomes, positive value matters, but less so than negative value; however, we do not always have reason to promote positive value. According to the deontological view, you are permitted but not required to bring about the best outcome given that it does not violate any deontological constraints. According to the satisficing version, you are permitted but not required to bring about an outcome that is more than sufficiently overall good (or, alternatively, contains a more than sufficiently amount of positive value). Even though creating the happy child would bring about the best consequences, as long as no deontological constraint is at stake, the parents are permitted but not required to create the child, according to the deontological view. According to the satisficing view, if not creating the child would bring about an outcome the value of which is sufficiently good (overall or in terms of positive value), the parents are permitted but not required to bring about the even better outcome in which there is an extra happy life.

The problem with the solution that invokes the deontological or satisficing version is that Asymmetry plays no role, or very little role, in explaining why the parent have no reason to create an extra happy child. The deontological and satisficing version would give the same verdict even if positive value would be given the same weight as negative value. What matters is just that there is a permission but no requirement to promote overall value, if no deontological constraint would be violated, or if there is an alternative outcome that is sufficiently good (overall or in terms of positive value).

The solution that invokes an asymmetry also at the normative level will avoid this 'idle wheel' problem. For here we have both a reason to promote positive value and a stronger reason to prevent negative value. However, this solution, as the author points out, will not work if we consider cases in which the child's life would be uniformly happy and thus not contain any negative wellbeing. The author is very cavalier about this shortcoming and claims such cases are unrealistic and that in the real world, the creation of the Happy Child would come at the cost of greater suffering, and such suffering would not be compensated by the increase in positive value.

It is true that cases in which a happy child is uniformly happy are unrealistic, but so are the cases Rozas discusses, since no other person beyond the parents and the child is supposed to be affected by the parents' choice. If the game we are playing invokes unrealistic examples, then it should be fine to invoke one in which the happy child would be uniformly happy, and here Rozas' solution will fail to deliver the intuitively right verdict. Our intuition that we lack reason to create happy people at the cost of wellbeing for already existing people does not vanish when we are told that the extra happy people will have uniformly happy lives. Indeed, the more general intuition is that we have no or little reason to create overall happy people but strong reason not to create overall unhappy people. This intuition cannot be explained by Rozas' asymmetric framework. That being said, I welcome his clear and bold approach to a difficult area in population ethics. It is an area rife 
with paradoxes and dilemmas and it is clear that no solution can be satisfactory in all respects. Some of our pet intuitions have to be abandoned.

\section{Reference}

Rozas, M. (2021). Two asymmetries in population and general normative ethics. Etikk $i$ Praksis - Nordic Journal of Applied Ethics, 15(1), 41-49. https://doi.org/10.5324/eip.v15i1.3860 